\title{
Rapid Identification of Allergen-Encoding cDNA Clones by Phage Display and High-Density Arrays
}

\author{
Rimantas Kodzius 1,2 , Claudio Rhyner 1 , Zoltan Konthur², Donald Buczek², Hans Lehrach², \\ Gerald Walter ${ }^{2}$ and , Reto Crameri ${ }^{*}$
}

${ }^{1}$ Swiss Institute of Allergy and Asthma Research (SIAF), Obere Strasse 22 CH -7270 Davos, Switzerland. ${ }^{2}$ Max Planck Institute for Molecular Genetics, Ihnestrasse 73, D-14195 Berlin, Germany

\begin{abstract}
We describe a high-throughput, quantitative technology for fast identification of all different clones present in selectively enriched phage surface-displayed cDNA libraries. The strategy is based on a combination of phage display and high-density arrays. To demonstrate the utility of the method cDNAs of Aspergillus fumigatus cloned into phagemid pJuFo were expressed on the tip of filamentous M13 phage and affinityselected on solid phase-immobilized serum IgE from allergic patients. Enriched phagemid libraries were amplified in bacteria, plated and arrayed into 384-well microtitre plates by robotic colony picking. cDNA inserts were amplified by high-throughput PCR and gridded onto high-density filter membranes. Filters were iteratively probed with randomly-sequenced inserts until all clones were identified. Eighty-one different sequences encoding IgE-binding proteins likely to cover a large part of the allergen repertoire of the mould were found. This approach represents a widely applicable method for rapid high-throughput identification of all individual cDNAs present in selectively enriched libraries.
\end{abstract}

Keywords: allergens, cDNA, phage display, high-density arrays, robotics.

Allergy represents a health problem in industrialized countries, where up to $20-30 \%$ of the population suffer from type I allergic symptoms like rhinitis, conjunctivitis or bronchial asthma [1]. A common hallmark of these diseases is the production of allergen-specific IgE raised against normally innocuous environmental allergens $[1,2]$. In sensitized atopic individuals, allergen exposure induces cross-linking of high-affinity FceRI receptor-bound IgE on effector cells and, thus, immediate release of anaphylactogenic mediators [2]. Although the mechanisms leading to allergic reactions are well understood $[3,4]$, our knowledge about the repertoire of molecular structures involved in the pathogenesis of allergic reactions is still fragmentary. This is mainly due to the complexity of many allergenic sources [5] and to the ability of the immune system of atopic individuals to mount $\operatorname{IgE}$ immune responses to a wide variety of molecular structures. Various allergens have been cloned and characterized using either direct PCR amplification or screening of bacteriophage $\lambda$ cDNA [6]. However, these approaches suffer from serious methodological limitations. While PCR requires before-hand sequence information, $\lambda$ expression libraries need to be screened on solid supports [7], hampering direct selection of clones expressing proteins of interest. In contrast, screening of cDNA libraries is greatly facilitated if it can be performed in solution by selective enrichment of relevant clones [8]. This requires a physical linkage between phenotype and genotype as in filamentous phage $[9,10]$ which display fusions to a coat protein on their surface while containing

*Address correspondence to this author at the Swiss Institute of Allergy and Asthma Research (SIAF), Obere Strasse 22 CH -7270 Davos, Switzerland; E-mail: crameri@siaf.unizh.ch the encoding DNA integrated into their single-stranded genome [8-11]. However, the display of cDNA libraries on filamentous phage is problematic. Efficient phage assembly requires the integrity of the carboxy-terminus of the most commonly used proteins pIII and pVIII [12]. Therefore, insertions of foreign proteins can only be tolerated at the amino-terminus $[11,13]$. Peptides coded by cDNAs generated by oligo dT-priming cannot be assembled in the phage coat by this approach since translation stop codons prevent the synthesis of $\mathrm{N}$-terminal fusions to the coat proteins [13]. To overcome these problems, several approaches including direct fusion of inserts to the carboxyl terminus region of gene VI [14], gene III and gene XIII of filamentous phage $[15,16]$, as well as the use of other phage systems $[17,18]$ have been proposed and recently reviewed $[16,19]$. We devised an indirect strategy, fusing the Jun zipper to the gene for phage protein III to enable coexpressed N-terminally Fos-decorated cDNA products to be efficiently displayed on the phage capsid via Jun-Fos interaction $[11,13]$. This technique has been widely used to clone allergens using immobilized serum IgE from allergic patients as ligand for enrichment of phage from surfacedisplayed cDNA libraries [20]. However, its full potential could not be unleashed because identifying all different clones present in an enriched library would have required a major sequencing effort of cDNA inserts. Instead, we have employed robotic-based devices [21], as developed for automated library handling in genomics $[22,23]$, for picking and high-density gridding of phage clones to rapidly catalogue redundant cDNA inserts. This efficient strategy enabled us to find rare clones in large, selectively enriched phage populations, and hence to define a vast variety of structurally different IgE-binding proteins form the mould Aspergillus fumigatus. 


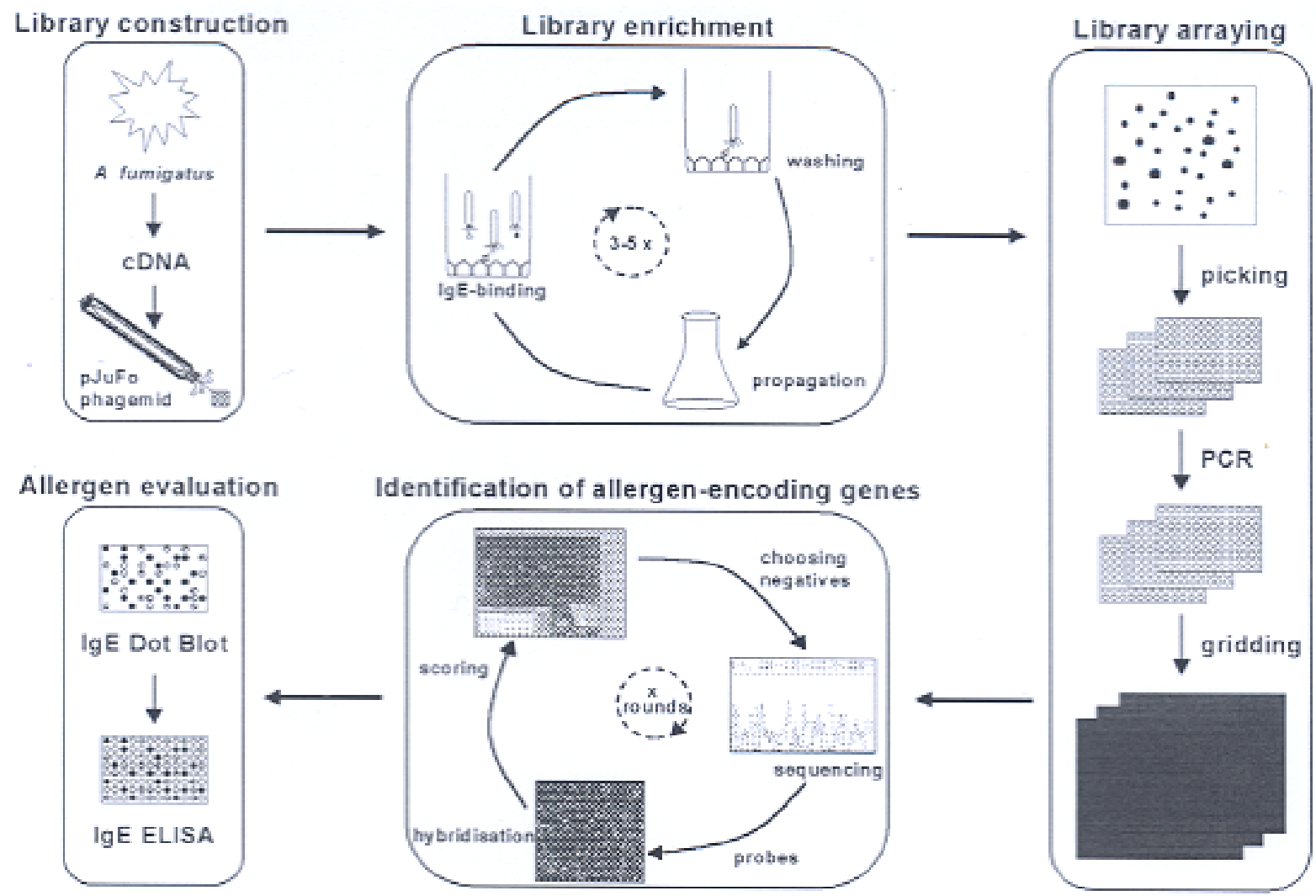

Fig. (1). Flow chart of the steps required for construction, selection and high throughput screening of cDNA libraries displayed on phage surface.

\section{EXPERIMENTAL}

\section{Construction of Phage Surface Displayed cDNA Libraries}

A previously described $\lambda$-ZapII cDNA expression library of A. fumigatus strain ATCC 42202 [24] was used as source of inserts to construct pJuFo phage surface displayed libraries $[11,13]$. Purified inserts prepared from in vivoexcised pBluescript phagemid by $\mathrm{XbaI}$ and $\mathrm{KpnI}$ digestion were ligated into $\mathrm{XbaI} / \mathrm{KpnI}$ digested pJuFo vector [20] and electroporated into E. coli XL-1 Blue (Stratagene, La Jolla, CA). Phage surface display libraries were generated by VCSM13 helper phage superinfection and titered as ampicillin resistant colonies [11]. The primary size of the library used for screening was $3 \times 10^{9}$ independent clones.

\section{Enrichment for IgE-Binding Phagemids by Biopanning}

IgE from pools of sera from individuals either suffering from A. fumigatus allergy or allergic bronchopulmonary aspergillosis [24, 28] was captured with solid phase bound monoclonal mouse anti-human IgE mAb TN-142 to produce a specific IgE-ligand surface as described [11]. After extensive washing with TBS ( $\mathrm{pH} 7.4), 0.5 \%$ Tween-20, $10^{11} \mathrm{cfu}$ of the phagemid library were added to each well and the plate incubated for $2 \mathrm{~h}$ at $37^{\circ} \mathrm{C}$. Binding phage were eluted and used to infect $2 \mathrm{ml}$ exponentially growing $E$. coli $\mathrm{XL1-Blue}$ cells to prepare phagemids for a further round of biopanning $[11-13,20]$.

\section{Automated Picking of Enriched Phagemid Libraries}

E. coli XL 1-Blue cells were infected with IgE-selected phagemid libraries, plated onto large square plates $(230 \mathrm{x}$ $230 \mathrm{~mm}$, Nunc) containing 2xYT agar, $100 \mu \mathrm{g} / \mathrm{ml}$ ampicillin and $2 \%$ glucose (2YT-AG) at a density of 30005000 colonies per plate and grown at $37^{\circ} \mathrm{C}$ overnight. Using a picking/gridding robot [25], 7 × 384 colonies per single enrichment were picked into 384 -well microtitre plates (Genetix, Christchurch, UK) containing 2xYT-AG medium supplemented with HMFM freezing mix $\left(0.4 \mathrm{mM} \mathrm{MgSO}_{4}\right.$, $1.5 \mathrm{mM} \mathrm{Na} 3$-citrate, $6.8 \mathrm{mM}\left(\mathrm{NH}_{4}\right)_{2} \mathrm{SO}_{4}, 3.6 \%$ glycerol, 13 $\mathrm{mM} \mathrm{KH} \mathrm{PO}_{4}, 27 \mathrm{mM} \mathrm{K}_{2} \mathrm{HPO}_{4}, \mathrm{pH}$ 7.0). After growth at $37^{\circ} \mathrm{C}$ overnight, plates were replicated into new microtitre plates using 384-pin replicating tools (Genetix) to produce working copies. The arrayed libraries were stored frozen at $-80^{\circ} \mathrm{C}$.

\section{High-Throughput PCR Amplification of Individual Clones}

cDNA inserts of all picked clones were amplified by PCR using a PTC-225 thermal cycler (Tetrad, MJ Research Inc., Watertown, MA) equipped with 384-well blocks. $30 \mu \mathrm{l}$ reaction mixes contained $0.2 \mathrm{mM}$ dNTP's, $0.24 \mu \mathrm{M}$ of each of the primers pJuFo5' (5'-TTCATCCTGGCGGCACACG3') and pJuFo3' (5'-CATGGGTTAAGCGGGATA-3'), 50 $\mathrm{mM}$ Tris-base, $50 \mathrm{mM} \mathrm{KCl}, 15 \mathrm{mM} \mathrm{MgCl} 2,0.1 \%$ Tween 20, 1.5 M betaine (Fluka, Buchs, Switzerland), $0.015 \mathrm{mM}$ cresol red (Sigma, St. Louis, MO) and $0.01 \mathrm{U} / \mu 1 \mathrm{Taq}$ 
polymerase. DNA templates were added as bacterial inoculates by transfer from 384-well microtitre plates using plastic replicators. Reaction mixes were heated to $94^{\circ} \mathrm{C}$ for 5 min and subjected to 30 amplification cycles consisting of $22 \mathrm{~s}$ at $94^{\circ} \mathrm{C}, 50 \mathrm{~s}$ at $57^{\circ} \mathrm{C}$ and $90 \mathrm{~s}$ at $72^{\circ} \mathrm{C}$, followed by a final extension at $72^{\circ} \mathrm{C}$ for $4 \mathrm{~min}$. Samples of $2 \mu 1$ of each PCR product were run on agarose gels to visualize successful amplification.

\section{Preparation of High Density Filters}

PCR products were gridded onto $222 \mathrm{~mm}$ x $222 \mathrm{~mm}$ Nylon filter membranes (Hybond- $\mathrm{N}^{+}$, Amersham, Buckinghamshire, UK) using a picking/gridding robot as described [25]. Spots were made by three consecutive pin hits transferring about $50 \mathrm{nl}$ of each PCR reaction mix. Clones were arranged in a duplicate pattern as blocks of $4 \mathrm{x}$ 4 surrounding ink guide dots. 2,688 (7 x 384) clones of each of the five enriched libraries were gridded, resulting in a total of 13,440 gridded clones. Even spotting and occasional gadget pin problems were visually judged by cresol red color intensity. After gridding, DNA was denatured by floating the filters on $0.4 \mathrm{M} \mathrm{NaOH}$ under gentle shaking for $2 \mathrm{~min}$ until spots turned bright red. Filters were neutralized in $5 \mathrm{x}$ SSC under gentle shaking until all spots turned yellow and air-dried on Whatman paper overnight. Dry filters were UVcrosslinked in a Stratagene crosslinker using the autolink setting, laminated [26] and stored at room temperature.

\section{Preparation of Hybridization Probes}

Labelled probes were prepared from cDNA inserts by PCR amplification incorporating digoxigenin (DIG-dUTP, Roche). $100 \mu 1$ reaction mixes containing $8.33 \mu \mathrm{M}$ DIGdUTP were set up and cycled as above, using a nested PCR strategy with a polyT23 oligonucleotide as back primer and 5'-GATCCCCCGGGCTGCAG-3' as forward primer.

\section{Filter Hybridizations}

Filter hybridizations using DIG-labeled PCR probes were performed as described [23]. Briefly, 15-100 $\mu 1$ probe and 2 $\mu 1$ each of oligonucleotide used for probe preparation as competitors $(100 \mathrm{pmol} / \mu \mathrm{l})$ were adjusted to $120 \mu \mathrm{l}$ total volume using TE ( $\mathrm{pH} \mathrm{8.0)}$ ). Mixtures were denatured at $98^{\circ} \mathrm{C}$ and incubated at $50^{\circ} \mathrm{C}-65^{\circ} \mathrm{C}$ for $1 \mathrm{~h}$ before they were dissolved in $7 \mathrm{ml}$ church buffer. Filters were pre-hybridized in church buffer at $65^{\circ} \mathrm{C}$ for $6 \mathrm{~h}$. Hybridization was performed at $65^{\circ} \mathrm{C}$ overnight. For detection, filters were washed by shaking in $20 \mathrm{mM} \mathrm{Na}_{2} \mathrm{HPO}_{4}(\mathrm{pH} 7.2), 0.1 \%$ SDS for 15 min and blocked in 5\% milk powder in PBS for $1 \mathrm{~h} .20 \mu \mathrm{l}$ anti-DIG alkaline phosphatase conjugate (Roche Diagnostics, Rotkreuz, Switzerland) dissolved in $100 \mathrm{ml}$ blocking buffer were added and filters incubated for $1 \mathrm{~h}$ at $20^{\circ} \mathrm{C}$. After washing twice for $30 \mathrm{~min}$ in PBS and for 10 min in $0.1 \mathrm{M}$ Tris $(\mathrm{pH} 9.5), 1 \mathrm{mM} \mathrm{MgCl} 2$, filters were incubated in a mixture of $80 \mathrm{ml} 0.1 \mathrm{M}$ Tris $(\mathrm{pH} 9.5), 1 \mathrm{mM}$ $\mathrm{MgCl}_{2}, 20 \mathrm{ml} 5 \mathrm{mM}$ attophos, and $2.4 \mathrm{M}$ diethylamine $(\mathrm{pH}$ $9.2, \mathrm{RT})$. Development of yellow fluorescence was followed under long-wave UV light, and a high-resolution CCD detection system was used to generate images of filters.

\section{Analysis of Hybridization Data}

Positive clones were scored using the image analysis package VisualGrid (GPC, Munich, FRG). Matrices of hybridization patterns were compared using the programme hybcompare (Buczek, unpublished).

\section{DNA Sequence Analysis}

PCR products were sequenced using dye-terminators on an automated sequencer (ABI-Perkin Elmer, Foster City, CA). Homology searches were performed with BLAST and the Genetics Computer Group program FASTA [27].

\section{Protein Expression and IgE-Binding Assay}

Putative allergen-encoding cDNAs were subcloned into the high-expression vector pQE-30 (Qiagen, Hilden, FRG) as $\mathrm{Bgl} \mathrm{II} \mathrm{or} \mathrm{Bam} \mathrm{H} 1$ and $\mathrm{KpnI}$ restricted inserts [20]. Proteins were expressed in the E. coli strain M15 [11] as Nterminal $[\mathrm{His}]_{6}$-fusions, purified in a single step by $\mathrm{Ni}^{2+}$ chelate affinity chromatography under denaturing conditions and refolded by dialysis against PBS pH $7.4[11,28]$. For IgE-specific dot blot analysis, the allergens were spotted onto Hybond-ECL nitrocellulose membranes (Amersham, Buckinghamshire, UK) and processed as described [28]. The IgE binding capacity of the proteins was determined by a standard solid-phase ELISA visualized by incubation with alkaline phosphatase-conjugated goat anti-mouse $\operatorname{IgG}$ antibody (Pierce, Rockford, IL) and 4-nitrophenyl phosphate as substrate [24].

\section{RESULTS}

\section{Enrichment of Phage Displaying A. Fumigatus Allergens}

Screening of an A. fumigatus phage surface displayed cDNA library with five different serum pools from patients suffering from A. fumigatus allergy during five rounds of biopanning, yielded large populations of phage putatively expressing IgE-binding proteins. Phage enrichment was monitored without screening by following the yield of phage eluted from each round of panning [11]. The IgE-enriched populations are likely to contain phage encoding the majority of the allergens needed to cover the whole repertoire of the mould (see below).

\section{Identification of Different Clones by Iterative Probing of High-Density Arrays}

2688 single colonies for each of the five phagemid populations enriched with different serum pools were robotpicked and arrayed onto medium-filled 384-well plates. After high-throughput PCR amplification, the inserts were high- 
density gridded in duplicate onto filter membranes to produce filters for hybridization. In a first round of screening, dioxigenin-labelled probes from the previously described A. fumigatus allergen [29] Asp f 1, Asp f 3, Asp f
4, Asp f 5, Asp f 6, Asp f 7, Asp f 8, Asp f 9, Asp f 10, Asp f 11, Asp f 12 and Asp f 13 were hybridized consecutively with the arrayed libraries. To prevent unspecific hybridization to vector sequences shared between

Table 1. Characteristics of the Cloned $A$. Fumigatus IgE-Binding Protiens

\begin{tabular}{|c|c|c|c|c|c|c|c|c|c|}
\hline Clone & ORF $^{a)}$ & Database match & Database match & Entry & Identity & Predicted & Sequence & Clone & IgE-Binding \\
\hline ID & & Organism & Gene & Accession No. & $(\%)$ & protein(aa) & Contig (bp) & frequency & capacity $^{\text {b), c) }}$ \\
\hline Aspf 09 & $\mathbf{P}$ & A. fumigatus & $\begin{array}{c}\text { Ferrioxamine } \mathrm{B} \\
\text { permease }\end{array}$ & AJ223327 & $(100)$ & 302 & 973 & 2055 & Ref. 28 \\
\hline Aspf 04 & $\mathrm{P}$ & A. fumigatus & Histone $\mathrm{H} 3$ & AJ001731 & $(100)$ & 286 & 861 & 669 & Ref. 29 \\
\hline Aspf 13 & $\mathrm{C}$ & A. fumigatus & Snodprot1 & AJ002026 & $(100)$ & 153 & 703 & 581 & Ref. 29 \\
\hline Aspf 01 & $\mathrm{C}$ & A. fumigatus & $\begin{array}{l}\text { Restrictocin } \\
\quad(\text { sarcin })\end{array}$ & M83781 & $(100)$ & 176 & 678 & 394 & Ref. 29 \\
\hline Aspf 07 & $\mathrm{P}$ & A. fumigatus & $\begin{array}{c}\text { Phytoene } \\
\text { desaturase (crtI) }\end{array}$ & AJ223315 & $(100)$ & 112 & 1058 & 281 & Ref. 29 \\
\hline Aspf 11 & $\mathrm{C}$ & A. fumigatus & $\begin{array}{l}\text { Cyclophilin-like } \\
\text { isomerase }\end{array}$ & AJ006689 & $(100)$ & 171 & 676 & 173 & Ref. 40 \\
\hline Aspf 03 & $\mathrm{C}$ & A. fumigatus & $\begin{array}{c}\text { Peroxisomal } \\
\text { membrane protein }\end{array}$ & U58050 & $(100)$ & 168 & 564 & 150 & Ref. 29 \\
\hline Aspf 06 & $\mathrm{C}$ & A. fumigatus & $\begin{array}{l}\text { Manganese } \\
\text { superoxide } \\
\text { dismutase }\end{array}$ & U53561 & (100) & 221 & 666 & 1 & Ref. 41 \\
\hline Aspf 05 & $\mathrm{C}$ & A. fumigatus & $\begin{array}{c}\text { Metalloprotease } \\
\text { MEP }\end{array}$ & Z30424 & $(100)$ & 388 & 1164 & 0 & Ref. 29 \\
\hline Aspf 08 & $\mathrm{C}$ & A. fumigatus & $\begin{array}{l}\text { Ribosomal protein } \\
\text { P2 }\end{array}$ & AJ224333 & $(100)$ & 111 & 336 & 0 & Ref. 42 \\
\hline Aspf 10 & $\mathrm{C}$ & A. fumigatus & Aspergillopepsin & X85092 & $(100)$ & 395 & 1528 & 0 & Ref. 29 \\
\hline Aspf 12 & $\mathrm{P}$ & A. fumigatus & $\begin{array}{c}\text { Heat shock protein } \\
\text { Hsp1 }\end{array}$ & U92465 & $(100)$ & 441 & 1326 & 0 & Ref. 43 \\
\hline Aspfxx2 & $\mathrm{P}$ & H. sapiens & $\begin{array}{c}\text { Put. zinc } \\
\text { transporter ZnT-3 }\end{array}$ & U76010 & (39) & 185 & 742 & 3636 & NT \\
\hline E1j01 & $\mathrm{P}$ & S. typhimurium & $\begin{array}{c}\text { Propionate } \\
\text { catabolism operon } \\
\text { PrpD }\end{array}$ & U51879 & (61) & 84 & 1209 & 2306 & + \\
\hline Aspf 17 & $\mathrm{P}$ & P. marneffei & $\begin{array}{l}\text { Cell wall antigen } \\
\text { (MP1) }\end{array}$ & AY007312 & (38) & 197 & 693 & 1601 & NT \\
\hline $\mathrm{U} 1 \mathrm{c} 01$ & $\mathrm{C}$ & E. nidulans & $\begin{array}{l}\text { Putative cell wall } \\
\text { protein }\end{array}$ & AJ011296 & (63) & 204 & 771 & 231 & +++ \\
\hline $\mathrm{A} 4 \mathrm{~b} 17$ & $\mathrm{P}$ & C. michiganensis & $\begin{array}{l}\text { Cellulase } \\
\text { precursor }\end{array}$ & AY007311 & $(45)$ & 202 & 781 & 164 & + \\
\hline $\mathrm{E} 3 \mathrm{c} 05$ & & ---- & --- & ---- & & 67 & 941 & 158 & NT \\
\hline $\mathrm{A} 2 \mathrm{k} 12$ & $\mathrm{P}$ & S. cerevisiae & $\begin{array}{l}\text { Elongation factor } \\
1 \text {-gamma }\end{array}$ & L01879 & (63) & 195 & 977 & 67 & ++ \\
\hline $\mathrm{E} 5 \mathrm{~m} 20$ & & --- & ---- & ---- & & 156 & 767 & 64 & ++++ \\
\hline $\mathrm{U} 1 \mathrm{j} 01$ & $\mathrm{P}$ & N. crassa & $\begin{array}{l}\text { Spore coat } \\
\text { precursor }\end{array}$ & AL451020 & (53) & 85 & 573 & 26 & ++ \\
\hline $\mathrm{A} 4 \mathrm{p} 05$ & $\mathrm{P}$ & G. hirsutum & EST & AI731559 & (45) & 70 & 1028 & 2 & ++ \\
\hline G1a18 & $\mathrm{P}$ & A. sativa & $\begin{array}{c}\text { Thaumatin-like } \\
\text { protein }\end{array}$ & L39774 & (32) & 147 & 705 & 143 & ++++ \\
\hline
\end{tabular}




\begin{tabular}{|c|c|c|c|c|c|c|c|c|c|}
\hline Clone & ORF ${ }^{a)}$ & Database match & Database match & Entry & Identity & Predicted & Sequence & Clone & IgE-Binding \\
\hline ID & & Organism & Gene & Accession No. & $(\%)$ & protein(aa) & Contig (bp) & frequency & capacity $^{\text {b), c) }}$ \\
\hline E1a02 & & $-\cdots$ & --- & --- & & 103 & 691 & 137 & + \\
\hline $\mathrm{U} 2 \mathrm{~b} 01$ & $\mathrm{P}$ & R. meliloti & FLIL protein & L49337 & (34) & 206 & 746 & 59 & +++ \\
\hline A4f19 & $\mathrm{C}$ & A. niger & EST & BE759128 & $(57)$ & 238 & 714 & 47 & + \\
\hline $\mathrm{U} 1 \mathrm{j} 04$ & $\mathrm{C}$ & P. chrysogenum & Thioredoxin & $\mathrm{X} 76120$ & $(55)$ & 139 & 584 & 47 & ++++ \\
\hline $\mathrm{G} 2 \mathrm{~b} 18$ & $\mathrm{C}$ & A. capsulatus & TSA1 & AF312927 & $(77)$ & 216 & 816 & 44 & ++ \\
\hline Aspf 02 & $\mathrm{C}$ & A. fumigatus & $\begin{array}{c}\text { Fibrinogen-binding } \\
\text { PRA1 }\end{array}$ & U56938 & $(100)$ & 299 & 1572 & 42 & Ref. 44 \\
\hline $\mathrm{G} 5 \mathrm{~m} 05$ & $\mathrm{P}$ & S. pombe & $\begin{array}{c}\text { Hypothetical } \\
\text { serine rich protein }\end{array}$ & AL023704 & (63) & 218 & 830 & 38 & ++++ \\
\hline U1n23 & & $-\cdots$ & --- & --- & & 183 & 724 & 33 & ++++ \\
\hline A1f17 & & $-\cdots$ & $-\cdots$ & $-\cdots$ & & 59 & 671 & 27 & NT \\
\hline U1a19 & $\mathrm{C}$ & S. pombe & Thioredoxin 2 & AJ003819 & $(54)$ & 143 & 638 & 17 & ++++ \\
\hline $\mathrm{A} 1 \mathrm{j} 21$ & $\mathrm{P}$ & S. cerevisiae & $\begin{array}{l}\text { PIR1 cell wall } \\
\text { protein }\end{array}$ & D13740 & (33) & 279 & 839 & 16 & ++ \\
\hline $\mathrm{G} 1 \mathrm{a} 04$ & $\mathrm{P}$ & S. cerevisiae & $\begin{array}{l}\text { Ribosomal protein } \\
\text { L2 }\end{array}$ & J03195 & (56) & 267 & 955 & 15 & ++++ \\
\hline $\mathrm{E} 3 \mathrm{~h} 02$ & & ---- & --- & ---- & & 90 & 807 & 13 & + \\
\hline $\mathrm{U} 2 \mathrm{j} 24$ & $\mathrm{P}$ & C. wickerhamii & $\begin{array}{l}\text { Putative beta- } \\
\text { glucosidase }\end{array}$ & U31091 & $(48)$ & 192 & 928 & 13 & +++ \\
\hline $\mathrm{E} 4 \mathrm{a} 21$ & & $-\cdots$ & --- & --- & & 20 & 752 & 10 & NT \\
\hline E1i01 & $\mathrm{P}$ & R. norvegicus & Mucin & U07615 & $(35)$ & 131 & 395 & 3 & NT \\
\hline $\mathrm{A} 2 \mathrm{~h} 06$ & $\mathrm{P}$ & M. anisopliae & $\begin{array}{c}\text { Putative } \\
\text { chitosanase }\end{array}$ & AJ293219 & (39) & 312 & 1080 & 8 & ++ \\
\hline $\mathrm{E} 4 \mathrm{j} 23$ & $\mathrm{P}$ & E. nidulans & SNAD (snaD) & AF070480 & $(71)$ & 193 & 854 & 6 & + \\
\hline E1k02 & $\mathrm{P}$ & S. pombe & $\begin{array}{c}\text { Cell wall } \\
\text { biogenesis protein }\end{array}$ & AB017617 & (32) & 407 & 1217 & 5 & + \\
\hline $\mathrm{E} 3 \mathrm{n} 12$ & & --- & --- & --- & & 129 & 586 & 5 & NT \\
\hline $\mathrm{E} 3 \mathrm{o} 02$ & & $-\cdots$ & --- & --- & & 123 & 705 & 5 & NT \\
\hline $\mathrm{E} 7 \mathrm{p} 12$ & $\mathrm{P}$ & A. muscaria & $\begin{array}{l}\text { Monosaccharide } \\
\text { transporter Mst-1 }\end{array}$ & Z83828 & (47) & 83 & 915 & 5 & ++++ \\
\hline E1d05 & $\mathrm{P}$ & A. oryzae & Alpha-glucosidase & D45179 & $(82)$ & 289 & 895 & 4 & ++++ \\
\hline $\mathrm{A} 2 \mathrm{e} 03$ & $\mathrm{P}$ & A. niger & EST & BE759952 & $(64)$ & 169 & 787 & 3 & + \\
\hline $\mathrm{C} 1 \mathrm{~h} 04$ & $\mathrm{P}$ & A. niger & EST & BE760005 & $(52)$ & 107 & 900 & 3 & ++++ \\
\hline E1e23 & $\mathrm{C}$ & S. pombae & $\begin{array}{l}\text { C-4 methyl sterol } \\
\text { oxidase }\end{array}$ & AL109832 & $(55)$ & 198 & 596 & 3 & ++++ \\
\hline E2c16 & $\mathrm{P}$ & S. cerevisiae & $\begin{array}{c}\text { Probable } \\
\text { membrane protein }\end{array}$ & U53881 & (56) & 238 & 823 & 3 & ++++ \\
\hline E2i04 & $\mathrm{P}$ & S. corieofaciens & $\begin{array}{c}\beta \text {-ketoacyl synthase } \\
\text { homologous }\end{array}$ & L20249 & (57) & 94 & 582 & 3 & NT \\
\hline E6j22 & & --- & --- & --- & & 55 & 1068 & 3 & ++ \\
\hline G6e10 & $\mathrm{P}$ & D. radiodurans & $\begin{array}{c}\text { Hypothetical } 31.1 \\
\text { kDa protein }\end{array}$ & AE001874 & $(35)$ & 112 & 891 & 3 & ++++ \\
\hline A7113 & $\mathrm{P}$ & A. niger & EST & BE760612 & (54) & 119 & 589 & 2 & NT \\
\hline
\end{tabular}


(Table 1) contd.....

\begin{tabular}{|c|c|c|c|c|c|c|c|c|c|}
\hline Clone & ORF $^{a)}$ & Database match & Database match & Entry & Identity & Predicted & Sequence & Clone & IgE-Binding \\
\hline ID & & Organism & Gene & Accession No. & $(\%)$ & protein(aa) & Contig (bp) & frequency & (capacityb), c) \\
\hline C7c21 & $\mathrm{P}$ & S. vulgaris & EST & BF628513 & (39) & 58 & 1075 & 2 & ++++ \\
\hline E1h15 & & ---- & ---- & ---- & & 39 & 763 & 2 & ++++ \\
\hline $\mathrm{E} 2 \mathrm{~g} 03$ & $\mathrm{P}$ & E. nidulans & $\begin{array}{c}\gamma \text {-amino-n-butyrate } \\
\text { transaminase }\end{array}$ & X15647 & (92) & 121 & 355 & 2 & ++++ \\
\hline E2p10 & $\mathrm{C}$ & S. cerevisiae & $\begin{array}{c}\text { Hypothetical } 16.7 \\
\text { kDa protein }\end{array}$ & Z48613 & (43) & 194 & 584 & 2 & + \\
\hline $\mathrm{E} 4 \mathrm{c} 01$ & $\mathrm{P}$ & A. niger & GPD & X99652 & $(91)$ & 213 & 639 & 2 & + \\
\hline $\mathrm{A} 2 \mathrm{o} 20$ & $\mathrm{P}$ & A. nidulans & HEX1 & AF239659 & $(78)$ & 164 & 767 & 1 & NT \\
\hline Asp f 18 & $\mathrm{P}$ & A. fumigatus & Serine protease & Y13338 & $(100)$ & 335 & 1030 & 1 & NT \\
\hline A5020 & $\mathrm{P}$ & H. sapiens & PPP1R6 & Y18206 & $(70)$ & 54 & 783 & 1 & + \\
\hline A6k05 & $\mathrm{P}$ & H. sapiens & $\begin{array}{l}\text { Hypothetical } \\
\text { protein }\end{array}$ & AC005764 & (48) & 41 & 913 & 1 & + \\
\hline C1i09 & $\mathrm{P}$ & S. solar & $\begin{array}{c}\text { 60S ribosomal } \\
\text { protein }\end{array}$ & AF045188 & (73) & 98 & 297 & 1 & +++ \\
\hline $\mathrm{C} 4 \mathrm{j} 04$ & & ---- & $-\cdots$ & ---- & & 28 & 741 & 1 & NT \\
\hline C4103 & $\mathrm{P}$ & E. nidulans & EST & AA783536 & $(38)$ & 192 & 708 & 1 & ++++ \\
\hline E3g15 & & --- & --- & --- & & 60 & 1022 & 1 & +++ \\
\hline E3h18 & $\mathrm{P}$ & S. pombe & $\begin{array}{c}\text { SP66 casein } \\
\text { kinase-1 (hhp1) }\end{array}$ & U10863 & (87) & 228 & 1019 & 1 & +++ \\
\hline E3p17 & $\mathrm{P}$ & N. crassa & EST & AI320412 & $(46)$ & 147 & 544 & 1 & ++++ \\
\hline E5i23 & $\mathrm{P}$ & N. crassa & MOM38 & X56883 & $(59)$ & 280 & 881 & 1 & + \\
\hline E5p16 & & ---- & --- & --- & & 147 & 550 & 1 & ++++ \\
\hline E6f06 & $\mathrm{P}$ & S. cerevisiae & $\begin{array}{c}\text { 6-phosphogluconate } \\
\text { dehydrogenase }\end{array}$ & U17155 & (76) & 172 & 717 & 1 & + \\
\hline E6g 13 & $\mathrm{P}$ & N. crassa & $\begin{array}{l}\text { Cytochrome } \\
\text { reductase }\end{array}$ & X02472 & $(60)$ & 214 & 645 & 1 & ++ \\
\hline E6k03 & & --- & --- & --- & & 14 & 556 & 1 & NT \\
\hline E6n01 & $\mathrm{C}$ & S. pombe & Cyclophilin & X53223 & $(78)$ & 166 & 587 & 1 & + \\
\hline E7b21 & & --- & ---- & ---- & & 207 & 621 & 1 & + \\
\hline G2111 & $\mathrm{P}$ & S. coelicolor & Monooxygenase & AL450450 & $(42)$ & 239 & 774 & 1 & +++ \\
\hline $\begin{array}{c}\text { G5e19 } \\
\text { Asp f } 22\end{array}$ & $\begin{array}{l}\mathrm{P} \\
\mathrm{P}\end{array}$ & $\begin{array}{l}\text { S. pombae } \\
\text { A. fumigatus }\end{array}$ & $\begin{array}{c}\text { Glutamine } \\
\text { synthetase } \\
\text { Enolase }\end{array}$ & $\begin{array}{c}\text { Z98977 } \\
\text { AF284645 }\end{array}$ & $\begin{array}{l}(76) \\
(100)\end{array}$ & $\begin{array}{l}143 \\
146\end{array}$ & $\begin{array}{l}609 \\
468\end{array}$ & $\begin{array}{l}1 \\
1\end{array}$ & $\begin{array}{c}++++ \\
\text { NT }\end{array}$ \\
\hline G6i10 & & ---- & ---- & ---- & & 15 & 614 & 1 & NT \\
\hline U4e19 & $\mathrm{P}$ & A. muscaria & $\begin{array}{l}\text { Monosacaride } \\
\text { transporter Mst-1 }\end{array}$ & Z83828 & (84) & 84 & 358 & 1 & + \\
\hline
\end{tabular}

a) Predicted reading frame: C, complete; P, partial. b),,+++++++++ and + refer to the OD of a standard positive serum pool of $>3,>2,>1$ and $>0.5$, respectively. c) NT: not tested

different inserts, oligonucleotides were used as competitors. High resolution images of the hybridized filters were compared using the program hybcompare. $4304(32.0 \%)$ of the 13440 clones analyzed hybridized with the known allergen sequences (Table 1). To prepare inserts for a second hybridization round, a randomly selected group of 38 hybridization negative clones from the first round were 5'sequenced yielding 10 new sequences. Consecutive hybridization of the arrayed library with labeled probes of these ten inserts allowed to identify 8255 (61.4\%) hybridization positive clones. Further sequencing of 41 hybridization negative clones yielded 20 so far unidentified sequences used for a third round of hybridization allowing the identification of $723(5.4 \%)$ hybridization positive clones. Finally, $158(1.2 \%)$ hybridization negative clones remained which were all PCR amplified and 5'-sequenced. 
84 of these clones gave no amplification product, indicating a failure rate of $0.6 \%$. Among the 74 remaining clones, 39 new sequences were found. Thus, after three rounds of hybridization including 12 already known sequences and 153 sequencing reactions $99.4 \%$ of the 13440 inserts could be assigned to a discrete sequence. The failure to detect positive clones for the known allergens Asp f 5, 8, 10 and 12 can be explained by the selection of sera used for the enrichment of the library aimed to avoid IgE raised against known allergens whenever possible, resulting in an under representation of these known sequences.

\section{Characterization of Aspergillus Fumigatus Allergens}

Inserts from all new clones identified by hybridization were PCR amplified and products 5'-sequenced to gather first hints about the putative molecular structures involved in eliciting A. fumigatus allergy. As expected from the screening of a cDNA library, not all sequences span complete open reading frames and six clones contain very short open reading frames of less than 50 amino acids probably representing protein fragments able to interact with $\operatorname{IgE}$ (Tab. 1). However, the sequence information allowed homology searches to be performed to tentatively assign the putative allergens to already known protein families. Interestingly, 10 of the new sequences show high homology to already described IgE-binding proteins including the $A$. fumigatus allergens Asp f 2, Asp f 18, Asp f 17 and Asp f 22 , whereas most of the other sequences show between 32 and $>90 \%$ identity to sequences deposited in the databases (Tab. 1). The high number of different putative allergens detected is in agreement with the extremely polyclonal $\operatorname{IgE}$ responses of atopic individuals to A. fumigatus exposure demonstrated by Western blot analysis of $A$. fumigatus extracts [30].

\section{Subcloning, Protein Expression and IgE-Binding Assays}

Inserts from 52 clones encoding new putative allergens were individually subcloned into the high level expression vector $\mathrm{pQE} 30$ to produce $\mathrm{N}$-terminal hexahistidine-tagged fusions. After cell lysis and single step purification over $\mathrm{Ni}^{2+}$-chelate affinity chromatography, proteins were dotblotted onto nitrocellulose membranes and probed for specific IgE-binding capacity as described elsewhere [28]. All 52 gene products showed strong signals, indicating the allergenic nature of the proteins (data not shown). IgE binding was first confirmed by titrable solid phase ELISA [18] against the serum pools used for selection (Tab. 1), followed by individual investigation of sera of ten patients suffering from allergic bronchopulmonary aspergillosis highly sensitized to the mould (data not shown). The results obtained unequivocally show the high specificity of the screening procedure for the isolation of IgE-binding proteins.

\section{DISCUSSION}

Using phage display technology, we have constructed a surface displayed cDNA library of the mould A. fumigatus, previously used for manual selection of phage enriched for their ability to interact with serum IgE from individuals allergic to A. fumigatus [11]. These phage have been shown to display allergenic molecules and as a consequence of the physical linkage between genotype and phenotype [13], to contain the genetic information responsible for their production integrated into the phage genome [9, 10]. Thus, the identification of clones expressing genes of interest in complex cDNA libraries displayed on phage surface is greatly facilitated by screening procedures based on selective enrichment $[9-13,20]$. However, the usual format based on biopanning and manual identification of clones is unlikely to allow a fast identification of all gene products enriched by complex ligands like patients sera [8]. For a fast and costeffective identification of all clones of an enriched cDNA library, high throughput screening technology is required. In a first step we have arrayed phagemid clones from selectively enriched libraries onto 384-well microtiter plates using robotic picking $[21,22]$. These high density arrays have the advantage that each clone has a unique position defined by the coordinates on the microtiter plate allowing unequivocal identification of each clone in later stages. For the screening procedure the inserts of all clones were amplified by high throughput PCR in 384-well blocks keeping the original coordinates of the arrayed clones constant, and products gridded at high density onto $22 \times 22 \mathrm{~cm}$ nylon filter membranes. Because each clone has a unique position, PCR products can be double spotted at high density and up to 15 identical filters can be generated simultaneously. The ability to create double spots and duplicate arrays help to eliminate false positives during hybridisation experiments and allows the screening of identical filters with different inserts to assign each hybridising signal to a discrete sequence and thus to the corresponding coordinates of the respective clone in the colony arrays. After three rounds of hybridisation with 42 different inserts, $98.3 \%$ of the 13440 inserts spotted could be unequivocally assigned to a discrete sequence. Of the remaining 158 clones 74 were PCR positive, and sequencing of the PCR products yielded 39 new sequences. Subcloning of 52 of the new inserts in a high level expression vector and subsequent serologic analysis of the gene products demonstrated that all proteins were able to bind serum IgE from patients allergic to A. fumigatus indicating that all selected clones code for allergenic molecules. Therefore we conclude that the technology described, based on phage display of cDNA libraries and robot-assisted handling of enriched phagemids permits a fast identification of all different clones present in the enriched library and drastically reduces the manual work involved. We were able to identify 81 different cDNAs encoding allergens of an Aspergillus fumigatus cDNA library displayed on the phage surface, enriched by selection on serum IgE of individuals sensitized to the mould. This example indicates the potential of the combination of phage surface display technology and high-throughput library screening for the isolation of cDNA clones from complex allergenic systems. High density arrays appear to be a versatile tool if applied to cDNA libraries displayed on phage surface for the fast establishment of catalogues of selectively enriched clones. As shown for the isolation of allergens, the strategy permits the use of heterogeneous ligands (like polyclonal serum IgE) to enrich clones from large cDNA libraries and can potentially be extended to 
study protein-protein interactions between clones present in two different libraries. The consequent application of functional enrichment and robotic-based screening will allow rapid generation of information in the field of functional genomics. However, it has to be mentioned that all display technologies based on biological systems will suffer from constraints limiting the system itself [31], and these apply also for the display of cDNA libraries [32]. Obvious limitations are related to the restricted codon usage of $E$. coli as host, the lack of post-translational modification [33] and to the toxicity of some gene products expressed in prokaryotic systems [34]. More serious limitations of phage display derive from the characteristics of the phage life cycle [35] and from the limited folding capacity of the host [36, 37]. In fact, filamentous phages are released from the host without breaking the integrity of the cell membrane [38]. As a consequence thereof, any cDNA gene product whose properties prevent it from crossing the membrane will not be secreted and, therefore, not be assembled into the phage coat. Even displayed proteins might not be selected using native interaction partners if they are not correctly folded [39], and it is well known that the folding capacity of the $E$. coli machinery is a limiting step in the expression of native like proteins [36, 37]. In spite of these limitations it is reasonable to assume that phage display technology, corroborated with rapidly developing technologies based on parallel handling of large numbers of clones as described in this work will play an essential role in generating information and reagents to facilitate progress in the field of life sciences.

\section{ACKNOWLEDGEMENTS}

Work at SIAF was supported by the Swiss National Science Foundation grants 31.50515.97 and 31.63381.00. R. Kodzius was supported by a fellowship from the Lithuanian Government Scholarship. We are grateful to Prof. $\mathrm{K}$. Blaser for his continuous support.

\section{REFERENCES}

[1] Casolaro, V., Georas, S.N., Song, Z., Ono, S.J. Curr. Opin. Immunol. 1966, 8, 796.

[2] Hopkin, J.M. Curr. Opin. Immunol. 1997, 9, 788.

[3] Sutton, B.J., Gould, H.J. The human IgE network. Nature 1993, 366, 421.

[4] Holowka, D., Baird, B. Annu. Rev. Biophys. Biomol. Struct. 1996, 25, 79.

[5] Pierson-Mullany, L.K., Jackola, D.R., Blumenthal, M.N., Rosenberg, A. Mol. Immunol. 2000, 37, 613.

[6] Chapman, M.D., Smith, A.M., Vailes, L.D., Arruda, L.K., Dhanaraj, V., Pomes, A. J. Allergy Clin. Immunol. 2000, 106, 409.

[7] Mierendorf, R.C., Precy, C., Yang, R.A. Meth. Enzymol. 1987, 152, 458 .
[9] Smith, G.P. Science 1985, 228, 1515.

[10] Kang, A.S., Barbas, C.F., Janda, K.D., Benkovic, S.J., Lerner, R.A. Proc. Natl. Acad. Sci. USA 1991, 88, 4363.

[11] Crameri, R., Jaussi, R., Menz, G., Blaser, K. Eur. J. Biochem. 1994, 226, 53.

[12] Kay, B.K., Winter, G., McCafferty, J. Phage display of peptides and proteins: a laboratory manual. Academic Press, San Diego, 1996.

[13] Crameri, R., Suter, M. Gene 1993, 137, 69.

[14] Jespers, L, Messens, J.H., de Keyser, A., Eeckhot, D., van den Brande, I., Gansermans, Q.G., Lauwereys, M.J., Vlasuk, G.P., Stranssens, P.E. Biotechnology 1995, 13, 378 .

[15] Fuh, G., Sidhu, S.S. FEBS Lett. 2000, 480, 231.

[16] Sidhu, S. S. Biomol. Eng. 2001, 18, 57.

[17] Zucconi, A., Dente, L., Santonico, E., Castagnoli, L., Cesareni, G. J. Mol. Biol. 2001, 307, 1329.

[18] Castagnoli, L., Zucconi, A., Quondam, M., Rossi, M., Vaccaro, P., Panni, S., Paoluzi, S., Santonico, E., Dente, L., Cesareni, G. Comb. Chem. High Throughput Screen. 2001, 4, 121.

[19] Rhyner, C., Kodzius, R., Crameri, R. Curr. Pharmacol. Biotechnol. 2002, 3, 13.

[20] Appenzeller, U., Blaser, K., Crameri, R. Ach. Immunol. Ther. Exp. 2001, 49, 19.

[21] Beugelsdijk, T.J. Automation Technologies for Genome Characterization. John Wiley \& Sons Inc, New York, 1997.

[22] Meier-Ewert, S., Maier, E., Ahmadi, A., Curtis, J., Lehrach, H. Nature 1993, 361, 375.

[23] Maier, E., Meier, E.S., Ahmadi, A.R., Curtis, J., Lehrach, H. J. Biotechnol. 1994, 12, 433.

[24] Moser, M., Crameri, R., Brust, E., Suter, M., Menz, G. J. Allergy Clin. Immunol. 1994, 93, 1.

[25] Lehrach, H., Bancroft, D., Maier, E. Interdisc. Scie. Rev. 1997, 22, 37 .

[26] Bancroft, D.R., Obrien, J.K., Guerasimova, A., Lehrach, H. Nucleic Acids Res. 1997, 25, 4160.

[27] Pearson, E.R. Genomics 1991, 11, 635.

[28] Moser, M., Crameri, R., Menz, G., Schneider, T., Dudler, T., Gmachl, M., Blaser, K., Suter, M. J. Immunol. 1992, 149, 454.

[29] Crameri, R. Contr. Microbiol. 1999, 2, 44.

[30] Baur, X., Weiss, W., Jarosch, B., Menz, G., Schoch, C., Schmitz-Schumann, M., Virchow, C. J. Allergy Clin. Immunol. 1989, 83, 839 .

[31] Santi, S., Caprone, S., Mennuni, C., Lahm, A., Tramontaani, A., Luzzago, A., Nicosia, A. J. Mol. Biol. 2000, 296, 497. 
[32] Rhyner, C., Weichel, M., Flückiger, S., Hemmann, S., Kleber-Janke, T., Crameri, R. Methods 2002 (in press).

[33] McVean, G.A., Hurst, G.D. J. Mol. Evol. 2000, 50, 264.

[34] Kranendok, M., Laires, A., Rueff, J., Estabrook, W.R., Verneulen, P.P. Crit. Rev. Toxicol. 2000, 30, 287.

[35] Russel, M. J. Mol. Biol. 1998, 279, 485.

[36] Baneys, F. Curr. Opin. Biotechnol. 1999, 10, 411.

[37] Gotterman, E.E., Hendrickson, W. A. Curr. Opin. Microbiol. 2000, 3, 197.

[38] Webster, R. In Phage Display of Peptides and Proteins. A Laboratory Manual. Kay, B.K, Winter, J., McCafferty, J. Eds. Academic Press Inc.: San Diego, CA, 1996, 1.

[39] Suter, M., Foti, M., Ackermann, M., Crameri, R. In Phage Display of Peptides and Proteins. A Laboratory Manual.
Kay, B.K, Winter, J., McCafferty, J. Eds. Academic Press Inc.: San Diego, CA, 1996, 195.

[40] Flückiger, S., Fijten, H., Whitley, P., Blaser, K., Crameri, R. Eur. J. Immunol. 2002, 32, 10.

[41] Crameri, R., Faith, A., Hemmann, S., Jaussi, R., Ismail, C., Menz, G., Blaser, K. J. Exp. Med. 1996, 184, 265.

[42] Mayer, C., Appenzeller, U., Seelbach, H., Achatz, G., Oberkofler, H., Breitenbach, M., Blaser, K., Crameri R. J. Exp. Med. 1999, 189, 1507.

[43] Kumar, A., Deddy, L.V., Schoanik, A., Kurup V.P. J. Allergy Clin. Immunol. 1993, 91, 1024.

[44] Kurup, V.P., Banerjee, B., Hemmann, S., Greenberger, P.A., Blaser, K., Crameri, R. Clin. Exp. Allergy 2000, 30, 988. 